\title{
INFLUENCIA DE BERMAS O CANALES EN LA ESTABILIDAD DE PILAS DE LIXIVIACIÓN
}

\author{
L.LUDENAA ${ }^{1}$, D. A. PARRA ${ }^{2}$, P. G. MENDOZA ${ }^{3}$
}

${ }^{1}$ Bachiller en Ingeniería Civil, Universidad Nacional de Ingeniería, Ingeniero Geotécnico en Medioambiente y Sustentabilidad, Ausenco Vector, Lima - Perú (lucas.ludena@gmail.com)

${ }^{2}$ Facultad de Ingeniería Civil, Universidad Nacional de Ingeniería, Lima - Perú, (denys.parra@anddes.com)

${ }^{3}$ Ingeniero Geotécnico Senior en Medioambiente y Sustentabilidad, Ausenco Vector, Lima Perú (pedro.mendoza@ausenco.com)

Resumen: La investigación está orientada al análisis de estabilidad de pilas de lixiviación mediante una nueva forma no convencional de estabilización consistente en el diseño de bermas (estructuras trapezoidales) y/o canales (depresiones) ubicadas en la interfase entre la pila de lixiviación y suelo de fundación. Se usó el modelo constitutivo Hardening-Soil (HS) para el cálculo de desplazamientos, y el método de reducción de parámetros vía elementos finitos (EF) para el cálculo de la estabilidad. Se revisaron los conceptos generales del HS y sus parámetros, los cuales fueron separados en dos grupos: parámetros calibrados según la curva esfuerzo - deformación de los ensayos triaxiales CD y parámetros asumidos convenientemente, de acuerdo a la curva de deformación volumétrica del mismo ensayo. Los materiales calibrados fueron: el mineral, el relleno estructural y el suelo de baja permeabilidad; seguidamente se modeló la pila de lixiviación mediante el programa de EF, PLAXISv8.2. Las variables consideradas fueron la posición de las estructuras de estabilización y sus dimensiones. Se analizó la estabilidad estática y pseudo-estática de la pila de lixiviación obteniéndose que el canal aumenta el factor de seguridad pseudo-estático en 4,25\%, independientemente de su longitud; la profundidad del canal es la variable más influyente, con una dimensión óptima de 1,2 m. Respecto a la superficie de falla, se obtuvo el volumen de mineral potencialmente mobilizado, incluyendo uno y dos canales, disminuyendo dicho volumen en $33 \%$ y $60 \%$, respectivamente. Los FS con equilibrio limite resultan aproximadamente $2 \%$ mayores que vía EF, las superficies de falla no fueron semejantes con ambas metodologías.

Palabras clave: Pila de Lixiviación, Hardening Soil, Elementos Finitos, Bermas y Canales, 


\section{INTRODUCCION}

El proyecto de una pila de lixiviación debe considerar el diseño de una instalación que proporcione adecuadas condiciones de estabilidad contra deslizamientos bajo condiciones estáticas y pseudo-estáticas. La estabilidad está influenciada principalmente por la resistencia al corte de la interfase entre el material de baja permeabilidad y la geomembrana, y muy raras veces por el mineral de la pila o la cimentación. Cotidianamente consultores especializados han proyectado bermas y canales en la base de pads de lixiviación con la finalidad de mejorar las condiciones de estabilidad de estas instalaciones y analizando estos efectos mediante métodos clásicos de equilibrio límite, observando efectivamente en la mayoría de los casos reales el incremento de los factores de seguridad. El criterio empírico utilizado ha sido "quitarle continuidad a la superficie de falla en bloque" a través de estas bermas y canales. Entonces el objetivo de la presente investigación es analizar la influencia de las estructuras, bermas o canales, en los factores de seguridad y en las superficies de falla de una pila de lixiviación considerando métodos rigurosos esfuerzo-deformación.

\section{SIMULACIÓN Y CALIBRACIÓN DE LOS DIFERENTES MATERIALES AL MODELO CONSTITUTIVO HARDENING SOIL}

Una manera de aproximar el comportamiento de un suelo es mediante el modelamiento de materiales. En la presente investigación se usó el modelo constitutivo Hardening Soil (HS) que está basado en criterios de la teoría de elasticidad mediante las leyes de Hooke [1] y la teoría de la plasticidad [2].

El modelo mencionado presenta o simula un estado de endurecimiento isotrópico, que significa que en el espacio triaxial donde en cada eje se ubica un esfuerzo correspondiente $\sigma_{1}$, $\sigma_{2}, \sigma_{3}$, el desarrollo del endurecimiento se da de manera uniforme manteniendo constante el centro y la forma de la superficie de fluencia.

HS es un modelo avanzado usado para la simulación del comportamiento de diferentes estados de suelos, tales como suelos blandos y suelos rígidos. Cuando se somete a una carga desviadora, el suelo muestra un aumento de la rigidez así como también un desarrollo de las deformaciones plásticas. Se aproxima mejor a los ensayos drenados; es decir, se ajusta más la forma de la hipérbola debido a que las presiones de poro no intervienen. El HS es muy superior al modelo primario hiperbólico por tres aspectos. Primero porque usa la teoría de la plasticidad en vez de la teoría de la elasticidad; segundo porque incluye la dilatancia del suelo; y tercero porque introduce un superficie de fluencia (cierre de superficie de fluencia sobre el eje de tensión isótropa p' del espacio de Cambridge) [3]. Los parámetros de este modelo son:

- Dependencia de la rigidez del estado de esfuerzos, $m$.

- Deformaciones plásticas debido a la carga primaria desviadora, $E_{50}^{\text {ref }}$.

- Deformaciones plásticas debido a la compresión primaria, $E_{\text {oed }}^{r e f}$.

- Elasticidad en la descarga/recarga, $E_{u r}^{r e f}$.

- Parámetros del modelo Mohr-Coulomb, $c, \phi, \psi$. 
La descripción de su simbología y del procedimiento para calibrarlos y todos los detalles con respecto a estos parámetros serán descritos en ítems adelante.

\subsection{Calibración al Modelo Hardenig Soil}

El proceso de determinación de los valores de los parámetros de un modelo constitutivo es llamado calibración. En este caso la calibración se llevará a cabo para los siguientes materiales, típicos en pilas de lixiviación: relleno estructural, suelo de baja permeabilidad y mineral de la pila.

La comprobación de la calibración se llevó a cabo vía la simulación del ensayo de compresión triaxial consolidado drenado mediante el programa de elementos finitos, en ese sentido, se tiene un modelo de dimensiones las mismas que en el ensayo de laboratorio o dimensiones cualquiera, pues el resultado es el mismo. De este modo se simuló equivalentemente para una muestra de 1 por 1 unidades de longitud. El esquema general para la simulación del triaxial es el siguiente:

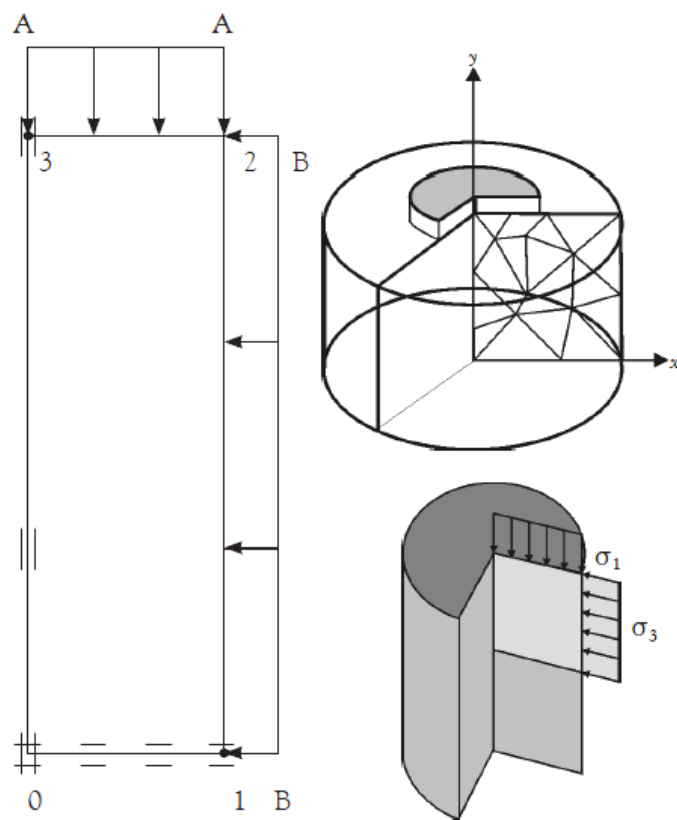

Figura 1. Modelamiento de la muestra de suelo en una simulación numérica por elementos finitos [4].

Tal como es el procedimiento del triaxial con los esfuerzos principal mayor y principal menor. Se agrupó el total de los parámetros en dos: el primero con los parámetros que serán calibrados con los ensayos disponibles y el segundo con los parámetros que serán asumidos debido a la no sensibilidad de la curva que se quiere ajustar. Entonces en ese sentido: 
Tabla1. Parámetros.

\begin{tabular}{|c|c|}
\hline Parámetros Calibrados & Descripción \\
\hline Peso Específico & $\begin{array}{l}\text { Encontrado de las propiedades físicas, en estado saturado y no } \\
\text { saturado. }\end{array}$ \\
\hline Cohesión, Ángulo de Fricción & $\begin{array}{l}\text { Se puede obener mediante los triaxiales } \mathrm{CD} \text {, } \\
\text { parámetros de falla. }\end{array}$ \\
\hline Ángulo de Dilatancia & $\begin{array}{l}\text { Se puede obener mediante los triaxiales } \mathrm{CD}, \\
\text { parámetros de falla (curva de deformación volumétrica). }\end{array}$ \\
\hline E_50^ref & $\begin{array}{l}\text { Encontrado de la curva esfuerzo deformación } \\
\text { correspondiente a la presión de referencia. }\end{array}$ \\
\hline $\mathrm{m}$ & $\begin{array}{l}\text { De la relación entre los valores de } \mathrm{E} \text { y la presión de } \\
\text { confinamiento - dependencia de la rigidez al estado de } \\
\text { esfuerzos. }\end{array}$ \\
\hline $\mathrm{p}^{\wedge} \mathrm{ref}$ & Presión de referencia, elegido el de $200 \mathrm{kPa}$. \\
\hline $\mathrm{K}_{\mathrm{x}}, \mathrm{K}_{\mathrm{y}}$ & $\begin{array}{l}\text { Permeabilidad en ambas direcciones y es medida con el ensayo } \\
\text { de conductividad hidráulica según ASTM D-5084. }\end{array}$ \\
\hline Reducción en la interfase (R_inter) & $\begin{array}{l}\text { Se encuentra de acuerdo a una razón de tangentes de ángulo de } \\
\text { fricción entre un material y una estructura, en este caso el la } \\
\text { tangente del ángulo de fricción del resultado del ensayo de corte } \\
\text { directo a gran escala y el ángulo de fricción del suelo de baja } \\
\text { permeabilidad. }\end{array}$ \\
\hline
\end{tabular}

Tabla2. Parámetros.

\begin{tabular}{ll}
\hline \multicolumn{1}{c}{ Parámetros Asumidos } & \multicolumn{1}{c}{ Descripción } \\
\hline E_oed^^ref & $\begin{array}{l}\text { Solo influye en la curva de variación volumétrica, que no } \\
\text { representa de manera correcta al comportamiento volumétrico } \\
\text { del material debido a que el procedimiento de lectura en el } \\
\text { triaxial CD podría ser imprecisa, además no se usará para la } \\
\text { investigación, por lo que su valorse podría estimar } \\
\text { aproximadamente. }\end{array}$ \\
E_ur^ref & $\begin{array}{l}\text { No es importante debido a que en la construcción de la pila no } \\
\text { hay proceso de descarga-recarga. }\end{array}$ \\
Ko & Se asume valores de 1-sen( $\phi$ '), según la bibliografia. \\
Rf & \\
Tracción & $\begin{array}{l}\text { Según las recomendaciones para el modelo HS se asume } \\
\text { típicamente un valor de } 0,9 .\end{array}$ \\
& $\begin{array}{l}\text { De acuerdo a las relaciones elásticas no presenta mayor } \\
\text { influencia en la sensibilidad de las curvas, además se tomó un } \\
\text { valor típico de } 0,3 \text { para todos los casos. }\end{array}$ \\
& el caso más real.
\end{tabular}




\subsubsection{Calibración del mineral}

$$
\phi^{\prime}, c^{\prime}, \psi^{\prime}
$$

Los valores de: Ángulo de fricción, cohesión y el ángulo de dilatancia del suelo se obtienen del ensayo triaxial consolidado - drenado $(\mathrm{CD})$ realizado.

\section{$E_{50}^{r e f} y \mathbf{m}$.-}

Estos parámetros se determinan a partir de los resultados de la curva esfuerzo deformación del ensayo triaxial consolidado drenado. El parámetro $E$ se determina según la gráfica de la relación hiperbólica en la cuerva esfuerzo - deformación del ensayo triaxial CD:

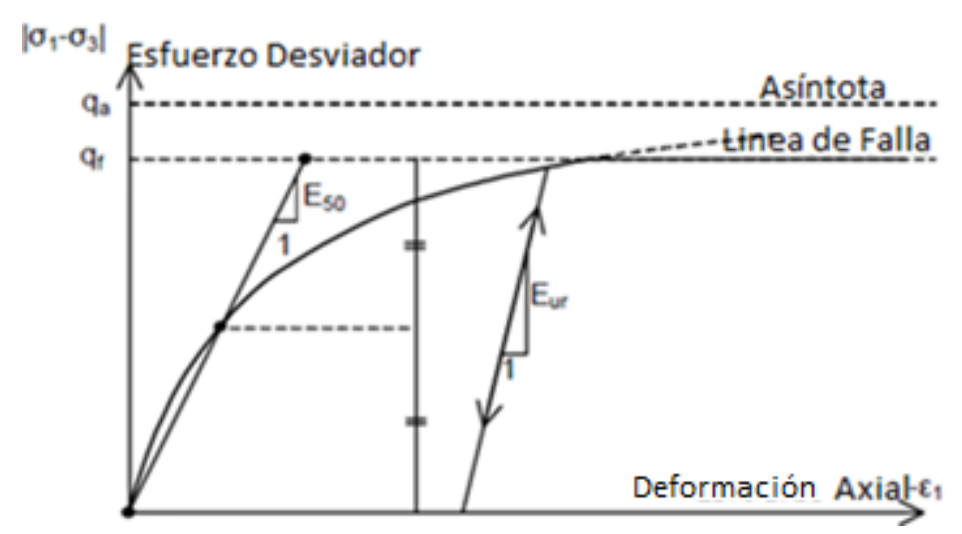

Figura 2. Curva esfuerzo - deformación de donde se obtiene $\mathrm{E}_{50}$ para cada presión de Confinamiento [3].

Se toma la presión de referencia de $200 \mathrm{kPa}$, entonces el $E_{50}^{r e f}$ es el $E_{50}$ correspondiente a la curva esfuerzo - deformación del ensayo triaxial consolidado drenado cuya presión de confinamiento es igual a la presión de referencia igual a $200 \mathrm{kPa}$.

Ahora con los tres valores de $E_{50}$ para cada presión de confinamiento se puede encontrar el valor de $m$, que estima la variación de la rigidez con respecto al estado de esfuerzos, entonces en el eje de las abscisas la presión de confinamiento y en el de las ordenadas el módulo de rigidez, se puede ajustar la curva según $A X^{B}$, donde $A$ es un número en unidades de presión y $B$ es el valor del parámetro m para las condiciones de rigidez dadas.

Por tanto:

Tabla3. Dependencia de E al Estado de Esfuerzos.

\begin{tabular}{cc}
\hline Pref $(\mathrm{kPa})$ & E50 $(\mathrm{kPa})$ \\
\hline 100 & 17625 \\
200 & 30900 \\
300 & 41860 \\
\hline
\end{tabular}




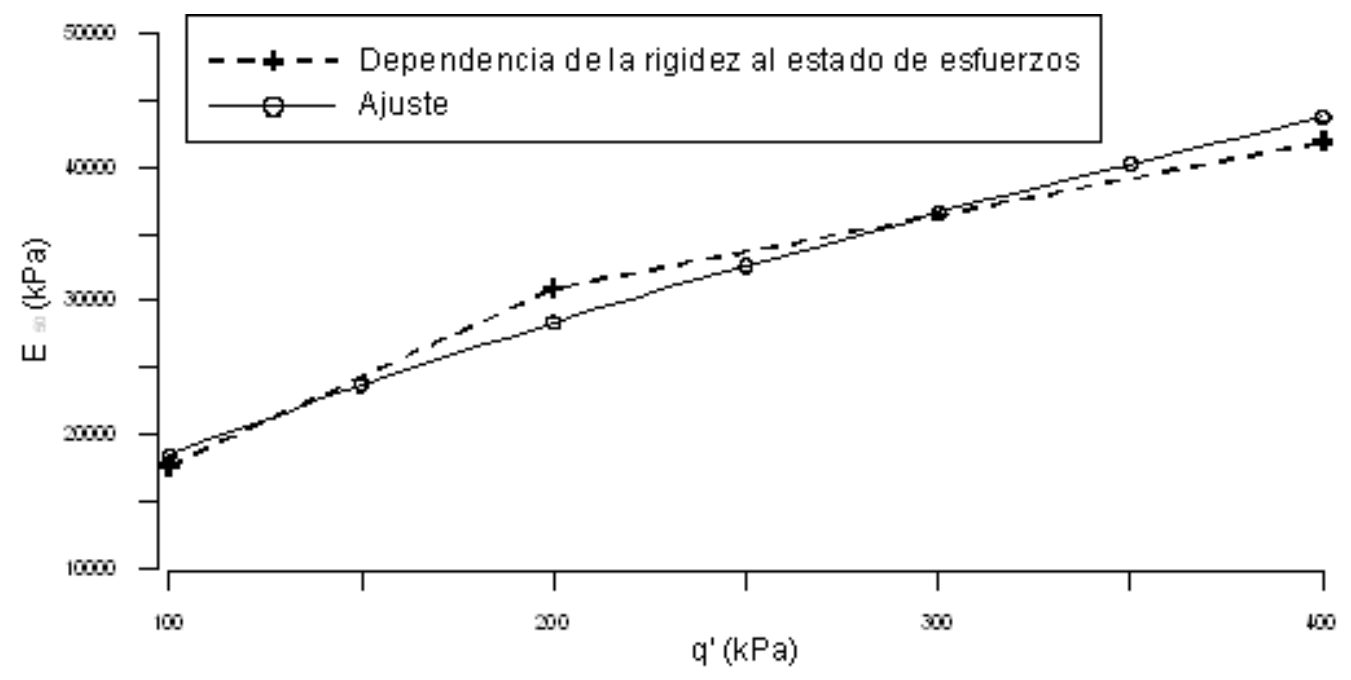

Figura 3. Dependencia de la rigidez al estado de esfuerzos.

Entonces se deduce que el valor de $m$ corresponde al valor de $B$ de la relación $A X^{B}$ correspondiente a la gráfica anterior; es decir $m=0,62$, un valor esperado para un material como el mineral, un GP sin compactación pues recordemos que el mineral se coloca al volteo, entonces un material granular de esas condiciones debería estar en ese orden de 0,5 a 0,65.

Permeabilidad en Kx, Ky.

Los coeficientes fueron obtenidos a partir de los ensayos de conductividad hidráulica realizados según la norma ASTM-D-5084. Se asume condiciones isotrópicas de la permeabilidad.

$$
\begin{aligned}
& \mathrm{k}_{\mathrm{x}}=3880 \mathrm{~m} / \text { día } \\
& \mathrm{k}_{\mathrm{y}}=3880 \mathrm{~m} / \text { día }
\end{aligned}
$$

Los parámetros restantes se obtienen de la siguiente forma: $\mathrm{k}_{\mathrm{o}}$ de la siguiente forma 1 -sen $\phi$ ', la relación de poisson para todos los casos es $v=0,30$ a excepción del suelo de cimentación en el cual se asumió $v=0,25, E_{50}^{\text {ref }}$ es el módulo tangente de un ensayo edométrico para una carga vertical igual a $\mathrm{P}^{\text {ref }}$, el $E_{u r}^{\text {ref }}$ del proceso de descarga - recarga en el ensayo triaxial. Como se explicó anteriormente algunos parámetros fueron asumidos debido a que no tienen mayor influencia sobre las deformaciones y por tanto tampoco en los desplazamientos.

Es importante indicar que no se presentan las gráficas de variación volumétrica versus deformación en sentido vertical porque, como se mencionó anteriormente, no se medirán variaciones de volumen.

Finalmente se tienen todos los parámetros del modelo HS para el mineral (Tabla4). Las curvas de calibración se presentan en la Figura4. 
Tabla4. Parámetros del Modelo del Mineral.

\begin{tabular}{ccl}
\hline Parámetros & Valores & \multicolumn{1}{c}{ Unidades } \\
\hline$\gamma_{\text {saturado }}$ & 18,1 & $\mathrm{kN} / \mathrm{m} 3$ \\
$\gamma_{\text {no-saturado }}$ & 17,1 & $\mathrm{kN} / \mathrm{m} 3$ \\
$\mathrm{c}^{\prime}$ & 0,0 & $\mathrm{kN} / \mathrm{m} 2$ \\
$\phi^{\prime}$ & 37 & $\circ$ \\
$\psi^{\prime}$ & 0 & $\circ$ \\
E_50^ref & 30000 & $\mathrm{kN} / \mathrm{m} 2$ \\
E_oed^ref & 20000 & $\mathrm{kN} / \mathrm{m} 2$ \\
$m$ & 0,62 & - \\
E_ur^ref & 90000 & $\mathrm{kN} / \mathrm{m} 2$ \\
v_ur & 0,3 & - \\
Pref & 200 & $\mathrm{kN} / \mathrm{m} 2$ \\
Ko & 0 & - \\
Kx & 3880 & $\mathrm{~m} / \mathrm{día}$ \\
Ky & 3880 & $\mathrm{~m} / \mathrm{día}$ \\
Rf & 0,9 & - \\
\hline
\end{tabular}

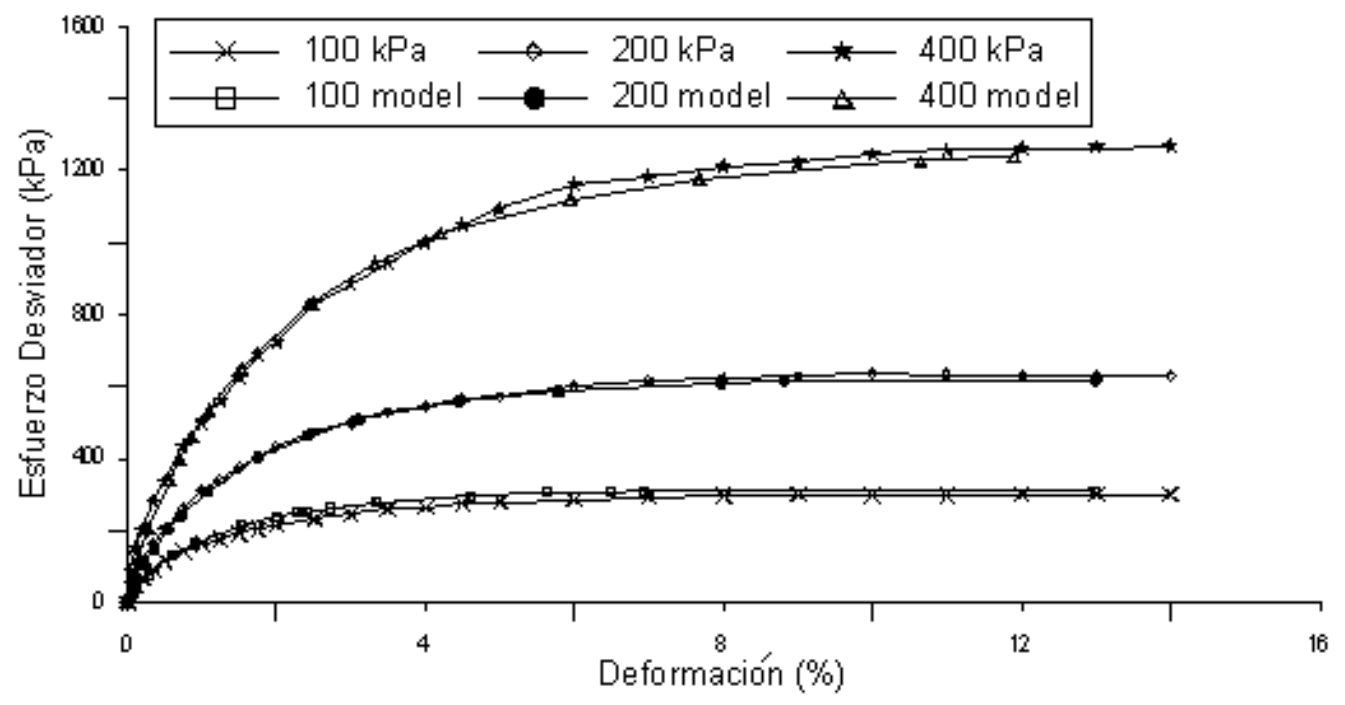

Figura 4. Gráficas experimental - modelo (esfuerzo - deformación).

No se colocará las gráficas de relaciones volumétrica versus deformación en sentido vertical porque como se mencionó anteriormente no se medirán variaciones de volumen.

\subsubsection{Calibración del relleno estructural}

Siguiendo el mismo procedimiento de calibración indicado anteriormente se presentan los resultados de los parámetros el relleno estructural en la Figura5. Se observa también una buena comparación con los resultados del ensayo triaxial CD. 
Tabla5. Parámetros del Modelo del Relleno Estructural.

\begin{tabular}{ccl}
\hline Parámetros & Valores & \multicolumn{1}{c}{ Unidades } \\
\hline$\gamma_{\text {saturado }}$ & 20,2 & $\mathrm{kN} / \mathrm{m} 3$ \\
$\gamma_{\text {no-saturado }}$ & 18,3 & $\mathrm{kN} / \mathrm{m} 3$ \\
' $^{\prime}$ & 14,0 & $\mathrm{kN} / \mathrm{m} 2$ \\
$\phi^{\prime}$ & 35,3 & $\circ$ \\
$\psi^{\prime}$ & 0 & $\circ$ \\
E_50^ref & 59350 & $\mathrm{kN} / \mathrm{m} 2$ \\
E_oed^ref & 50000 & $\mathrm{kN} / \mathrm{m} 2$ \\
$m$ & 0,31 & - \\
E_ur^ref & 178050 & $\mathrm{kN} / \mathrm{m} 2$ \\
v_ur & 0,3 & - \\
Pref & 200 & $\mathrm{kN} / \mathrm{m} 2$ \\
Ko & 0 & - \\
Kx & $3,00 \mathrm{E}-04$ & $\mathrm{~m} / \mathrm{día}$ \\
Ky & $3,00 \mathrm{E}-04$ & $\mathrm{~m} / \mathrm{día}$ \\
Rf & 0,9 & - \\
\hline
\end{tabular}

Las gráficas del esfuerzo - deformación del modelo y la experimental se presentan a continuación.

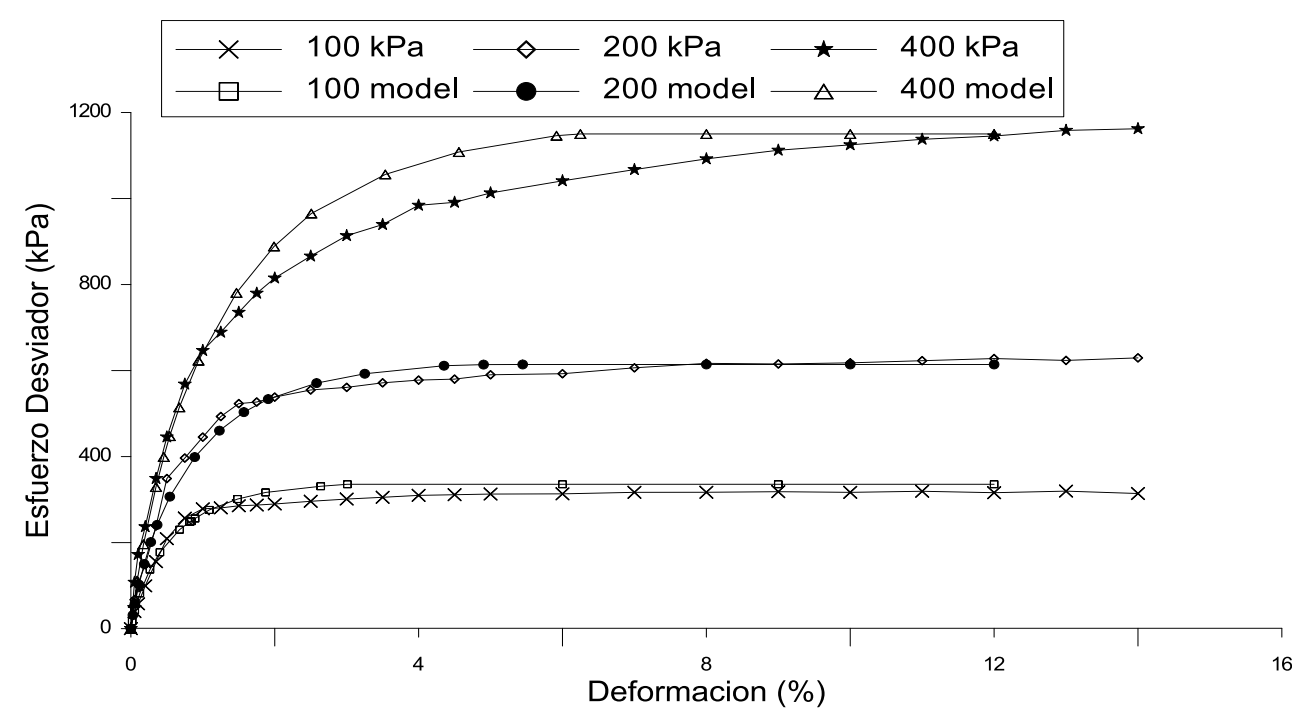

Figura 5. Gráficas experimental - modelo (curva esfuerzo - deformación).

\subsubsection{Calibración del suelo de baja permeabilidad}

Si bien en una pila de lixiviación la estabilidad está bastante influenciada por la resistencia de la interfase suelo-geomembrana, la calibración de la capa de suelo de baja permeabilidad, de manera similar que para los casos anteriores, se hace necesaria debido a que el suelo de cimentación ha sido modelado como un material elástico; de este modo, es posible aplicar, 
para el cálculo de la estabilidad, el concepto de reducción de los parámetros de resistencia de la interfase, el cual es presentado a continuación

\section{Reducción de la Interfase ( $\left.\mathbf{R}_{\text {inter }}\right)$--}

Se calibra con el ensayo de corte directo a gran escala (ASTM D-5321B). Es importante mencionar que la geomembrana usada en el ensayo fue LLDPE 2.0mm (lado texturado). Los resultados del ensayo fueron:
Adhesión
$=15 \mathrm{kPa}$
Ángulo de fricción
$=21,7^{\circ}$

Entonces la reducción de la resistencia entre el suelo de baja permeabilidad y la geomembrana es la razón de tangentes del ángulo de fricción entre estos dos materiales, y también la razón entre las cohesiones, resultando para este caso:

$\frac{\tan (\phi \text { corte directo a gran escala })}{\tan (\phi \text { suelo de baja permeabilidad })}=\frac{\tan \left(21,7^{\circ}\right)}{\tan \left(36,7^{\circ}\right)}=0,534$

De este modo $R_{\text {inter }}=0,534$, luego de obtener este parámetro el procedimiento para obtener los demás es el mismo que en los casos anteriores; por tanto:

Tabla6. Parámetros del Modelo del Suelo de Baja Permeabilidad.

\begin{tabular}{|c|c|c|}
\hline Parámetros & Valores & Unidades \\
\hline$\gamma_{\text {saturado }}$ & 20,6 & $\mathrm{kN} / \mathrm{m} 3$ \\
\hline$\gamma_{\text {no-saturado }}$ & 18,1 & $\mathrm{kN} / \mathrm{m} 3$ \\
\hline$c^{\prime}$ & 14 & $\mathrm{kN} / \mathrm{m} 2$ \\
\hline$\phi^{\prime}$ & 36,7 & 。 \\
\hline$\psi^{\prime}$ & 0 & $\circ$ \\
\hline E_50^ref & 89555 & $\mathrm{kN} / \mathrm{m} 2$ \\
\hline E_oed ${ }^{\wedge}$ ref & 80000 & $\mathrm{kN} / \mathrm{m} 2$ \\
\hline$m$ & 0,38 & - \\
\hline E_ur^ref & 268665 & $\mathrm{kN} / \mathrm{m} 2$ \\
\hline v_ur & 0,3 & - \\
\hline Pref & 200 & $\mathrm{kN} / \mathrm{m} 2$ \\
\hline Ko & 0 & - \\
\hline $\mathrm{Kx}$ & $2,70 \mathrm{e}-4$ & m/día \\
\hline Ky & $2,70 \mathrm{e}-4$ & m/día \\
\hline $\mathrm{Rf}$ & 0,9 & - \\
\hline
\end{tabular}

Las gráficas del esfuerzo - deformación del modelo y la experimental se presentan a continuación. 


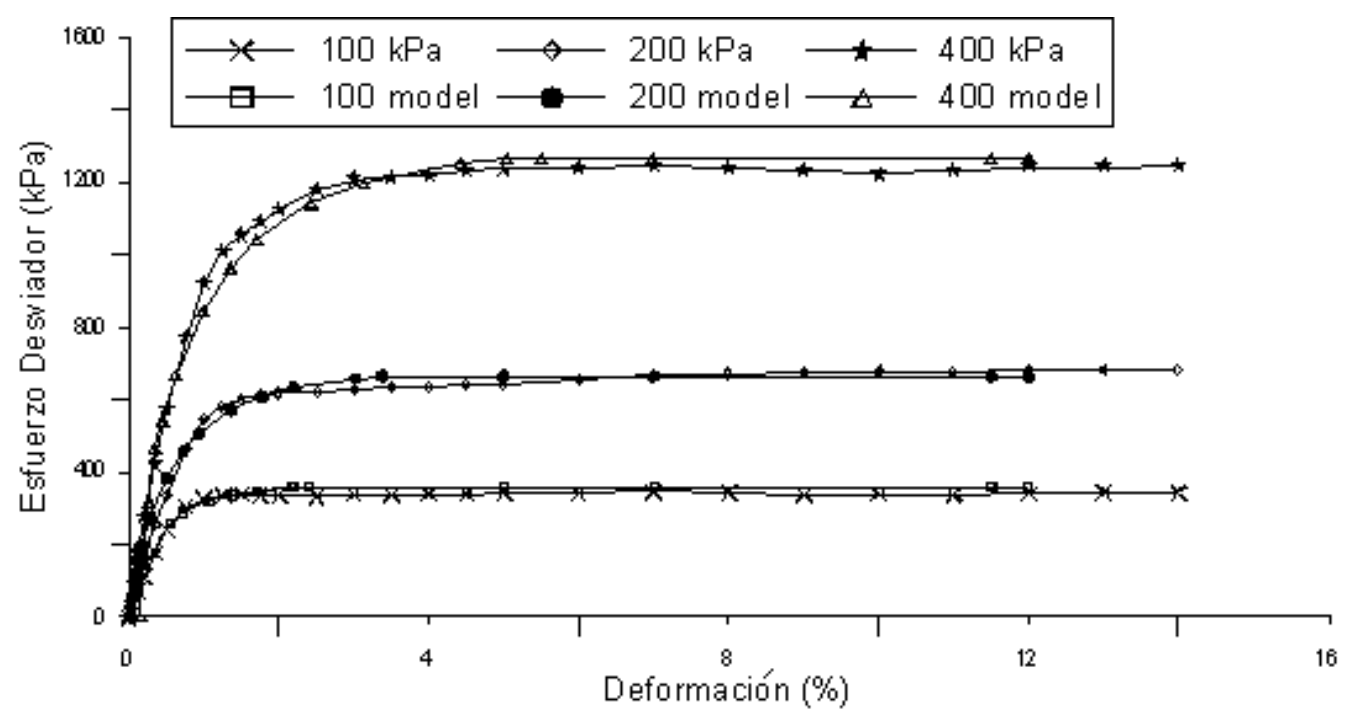

Figura 6. Gráficas experimental - modelo (curva esfuerzo - deformación).

\subsubsection{Suelo de cimentación}

La superficie de falla para el análisis de estabilidad de la pila se desarrolla básicamente en la interfase entre el suelo de baja permeabilidad y la geomembrana, asumiendo que el suelo de cimentación es suficientemente competente; por lo tanto, el suelo de cimentación no interviene en el análisis, debido a lo cual las propiedades de este material solo han sido asumidas para el análisis numérico considerando un material granular, y son presentados en la Tabla 7 [5].

Tabla7. Parámetros del Modelo del la Cimentación.

\begin{tabular}{ccl}
\hline Parámetros & Valores & \multicolumn{1}{c}{ Unidades } \\
\hline$\gamma_{\text {saturado }}$ & 25,5 & $\mathrm{kN} / \mathrm{m} 3$ \\
$\gamma_{\text {no-saturado }}$ & 24,5 & $\mathrm{kN} / \mathrm{m} 3$ \\
$\mathrm{E}$ & $1,96 \mathrm{e}+7$ & $\mathrm{kN} / \mathrm{m} 2$ \\
$v$ & 0,25 & - \\
$\mathrm{Kx}$ & $3,00 \mathrm{e}-4$ & $\mathrm{~m} / \mathrm{día}$ \\
$\mathrm{Ky}$ & $3,00 \mathrm{e}-4$ & $\mathrm{~m} / \mathrm{día}$ \\
\hline
\end{tabular}

\section{ESTABILIZACIÓN EN PILAS DE LIXIVIACIÓN}

El cálculo del factor de seguridad (FS) es mediante el procedimiento de reducción de parámetros. De este modo el FS es definido como el factor por el cual los parámetros de resistencia del suelo deben ser divididos con el objetivo de alcanzar las condiciones de falla (parámetros de falla); es decir los parámetros $C_{f}^{\prime} \mathrm{y} \phi_{f}^{\prime}:$ [6]

$$
\mathrm{c}_{\mathrm{f}}^{\prime}=\frac{\mathrm{c}^{\prime}}{\mathrm{SRF}} \text {. }
$$




$$
\phi_{\mathrm{f}}^{\prime}=\tan ^{-1}\left(\frac{\tan \phi^{\prime}}{\mathrm{SRF}}\right)
$$

\subsection{Pila de lixiviación analizada}

Se analizó para el caso particular de una pila de lixiviación ubicada en el departamento de Cajamarca (región nor-oriental del Perú) con altitud entre 3800 y 4000 msnm. En la Figura7 se muestra la sección típica de esta pila, cuya altura medida entre la cresta y el pie es de $130 \mathrm{~m}$. El talud de la pila es de 2,5H:1V y presenta alturas de banco de $10 \mathrm{~m}$.

Cabe aclarar que para calcular el factor de seguridad de una estructura como la de una pila de lixiviación sería suficiente considerando diferentes simplificaciones y no incurriendo en un error significativo con ahorro de tiempo de cómputo considerable. De este modo podría no considerarse el proceso de colocación de cada capa de mineral de altura $10 \mathrm{~m}$ colocando todo el material en una sola fase de cálculo. El suelo de baja permeabilidad podría simplificarse también pues no se estaría midiendo ni deformaciones ni esfuerzos en él, solo para el caso del cálculo de estabilidad. Adicionalmente se pudo haber usado un modelo constitutivo más básico como el modelo Mohr-Coulomb; sin embargo, se buscó la manera de representar más realmente el comportamiento esfuerzo - deformación de los materiales para obtener resultados de desplazamientos más cercanos a la realidad. Adicionalmente se consideró el conjunto de interfase de la pila de lixiviación por separado: suelo de baja permeabilidad y geomembrana, además de un material de relleno estructural; por lo que, la única simplificación con respecto a los materiales fue con la fundación en la que si se consideró sus parámetros tal y como fue expuesto en la sección anterior.

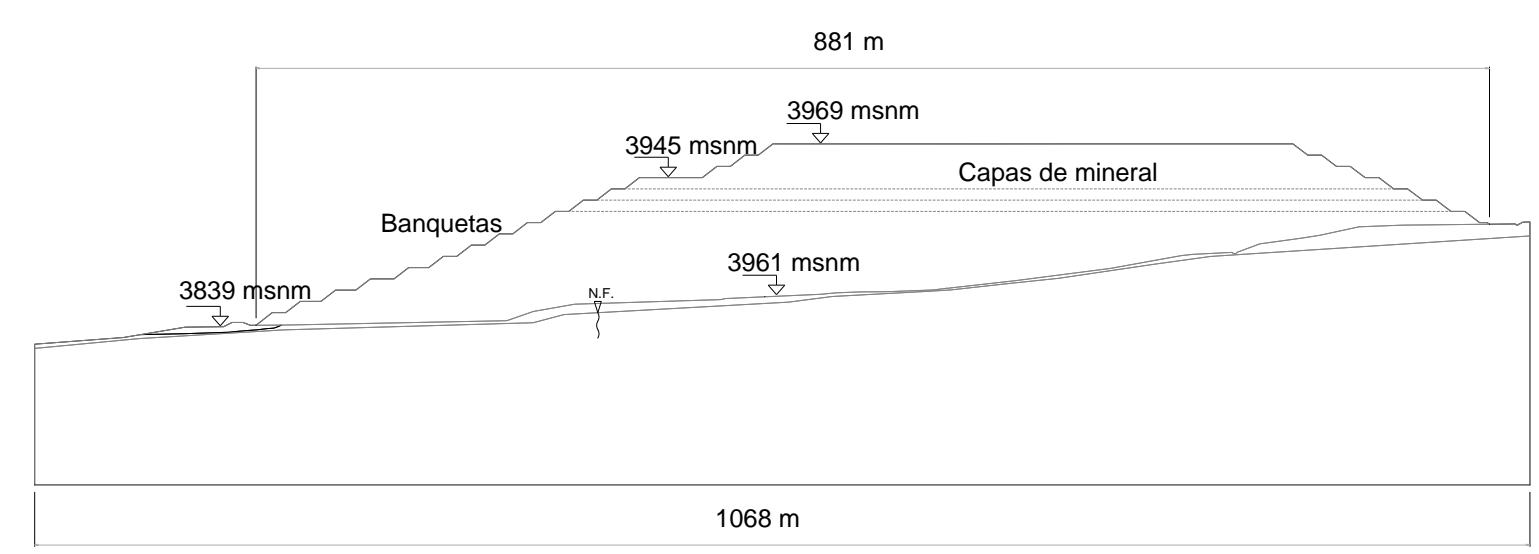

Figura 7. Sección de la pila analizada.

\subsubsection{Bermas y canales}

A continuación se presenta el modelo geométrico de las bermas y canales usados, de dimensiones estándares. 

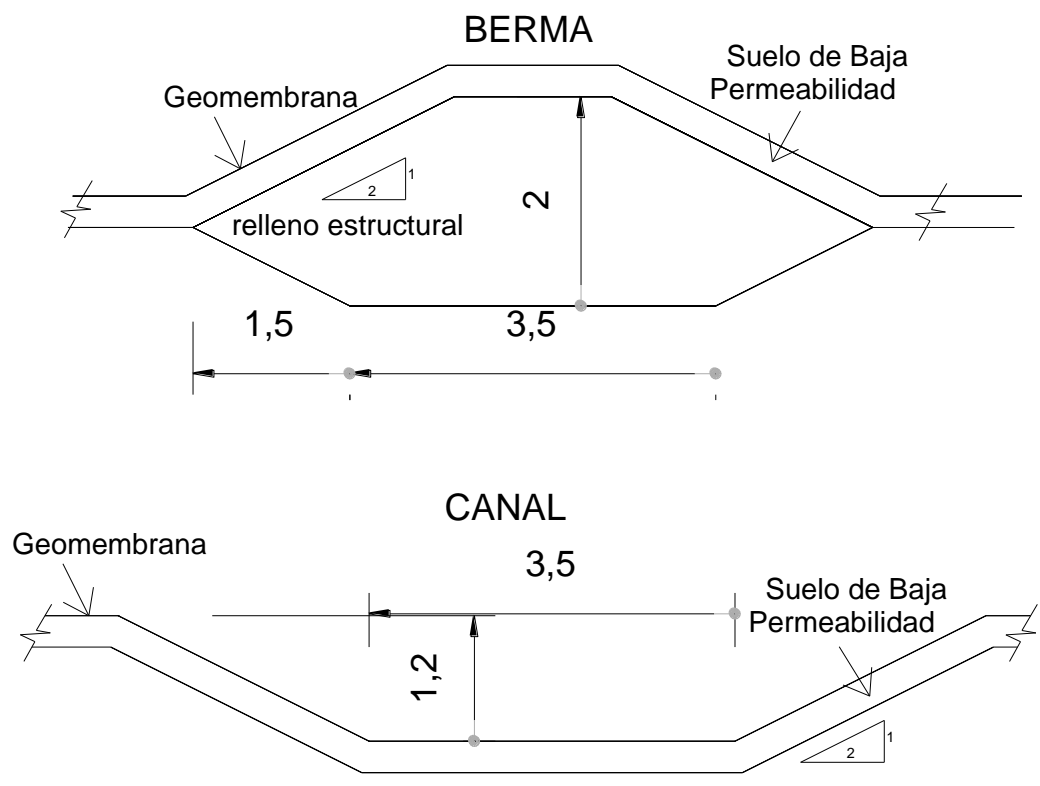

Figura 8. Dimensiones estándar de la berma y canal con suelo de baja permeabilidad de espesor $0,30 \mathrm{~m}$.

\subsection{Análisis estático y pseudo-estático}

\subsubsection{Variación de la posición de los canales y bermas}

En este ítem para la estabilidad estática se hizo variar la posición del canal o de la berma tal como se muestra en la Tabla8.y en la Figura9.

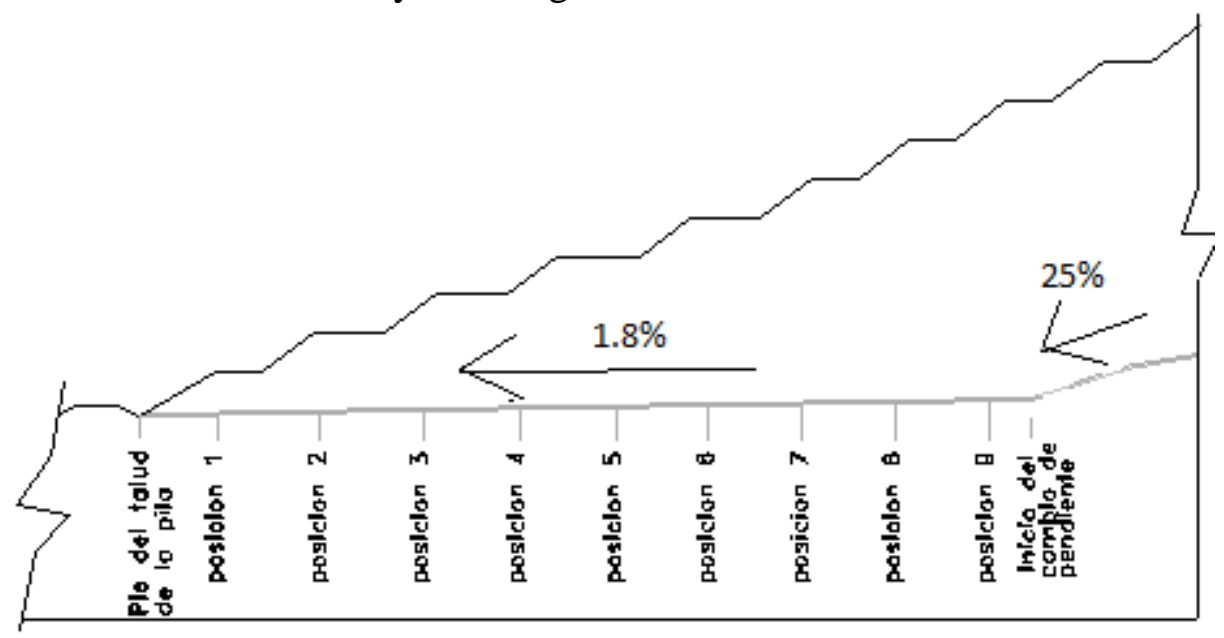

Figura 9. Posiciones relativas al pie del talud del mineral. 
Tabla8. Posiciones relativas de berma o canal respecto al pie del talud.

\begin{tabular}{cc} 
Posiciones & $\begin{array}{c}\text { Posiciones } \\
\text { relativas al pie } \\
\text { del talud (m) }\end{array}$ \\
\hline 1 & 16,0 \\
2 & 37,2 \\
3 & 58,2 \\
4 & 78,0 \\
5 & 97,9 \\
6 & 116,9 \\
7 & 136,1 \\
8 & 155,2 \\
9 & 174,4 \\
\hline
\end{tabular}

En la Tabla9 y Tabla10 se muestran los FS para cada posición de berma o canal de acuerdo a la Tabla8, en condiciones estáticas y pseudo-estáticas de carga respectivamente. Se conoce que el $\mathrm{FS}_{\text {estático }}=1,697$, y $\mathrm{FS}_{\text {pseudo-estático }}=1,058$ (el coeficiente sísmico fue 0,15).

Tabla9. FS en las diferentes posiciones relativas e incremento en porcentaje respecto al FS inicial (Estático)

\begin{tabular}{ccccc}
\hline $\begin{array}{c}\text { Ubicación relativa } \\
\text { respecto } \\
\text { al pie del latud }\end{array}$ & $\begin{array}{c}\text { Abscisa } \\
(\mathrm{m})\end{array}$ & $\begin{array}{c}\text { FS } \\
\text { Berma }\end{array}$ & $\begin{array}{c}\text { Incremento del FS (\%) } \\
\text { Berma }\end{array}$ & Canal \\
\hline 1 & 16,0 & 1,667 & $-1,74$ & $-2,18$ \\
2 & 37,2 & 1,679 & $-1,07$ & $-0,91$ \\
3 & 58,2 & 1,691 & $-0,36$ & $-0,31$ \\
4 & 78,0 & 1,699 & 0,12 & 0,22 \\
5 & 97,9 & 1,706 & 0,50 & 0,97 \\
6 & 116,9 & 1,712 & 0,85 & 1,61 \\
7 & 136,1 & 1,713 & 0,94 & 1,98 \\
8 & 155,2 & 1,716 & 1,11 & 2,22 \\
9 & 174,4 & 1,720 & 1,36 & 2,56 \\
\hline
\end{tabular}

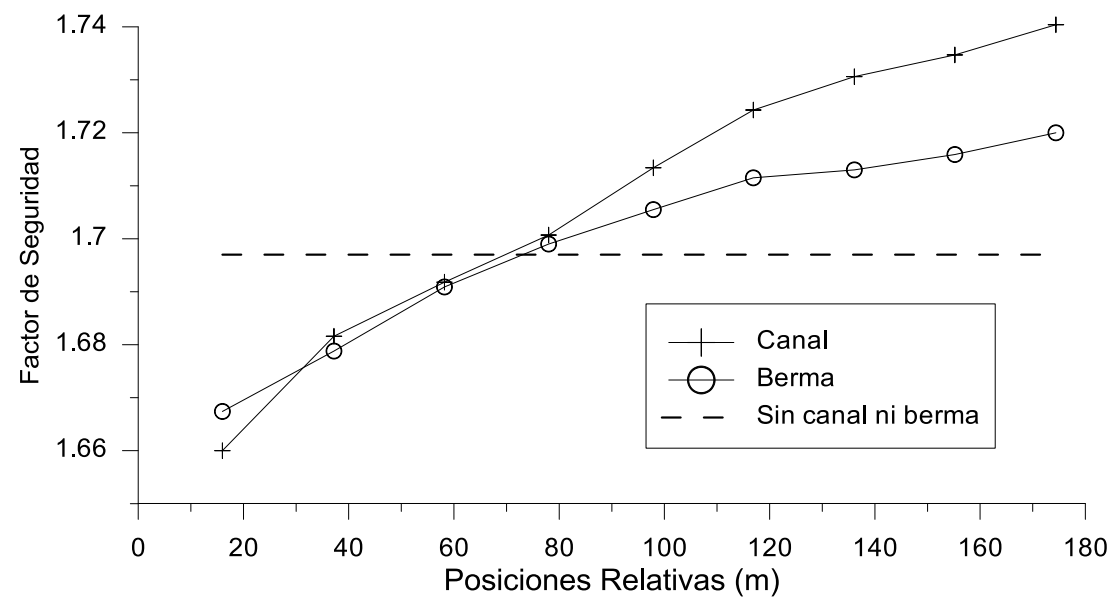

Figura 10. Factor de seguridad (estático) respecto a la posición. 
Tabla10. FS en las diferentes posiciones relativas e incremento en porcentaje respecto al FS inicial (Pseudo - Estático)

\begin{tabular}{ccccc}
\hline $\begin{array}{c}\text { Ubicación relativa } \\
\text { respecto } \\
\text { al pie del latud }\end{array}$ & $\begin{array}{c}\text { Abscisa } \\
(\mathrm{m})\end{array}$ & $\begin{array}{c}\text { FS } \\
\text { Berma }\end{array}$ & $\begin{array}{c}\text { Incremento del FS (\%) } \\
\text { Berma }\end{array}$ & Canal \\
\hline 1 & 16,0 & 1,050 & $-0,77$ & $-0,57$ \\
2 & 37,2 & 1,064 & 0,55 & 0,63 \\
3 & 58,2 & 1,072 & 1,29 & 1,40 \\
4 & 78,0 & 1,077 & 1,75 & 2,04 \\
5 & 97,9 & 1,084 & 2,46 & 2,93 \\
6 & 116,9 & 1,086 & 2,62 & 3,25 \\
7 & 136,1 & 1,089 & 2,91 & 3,75 \\
8 & 155,2 & 1,088 & 2,80 & 3,69 \\
9 & 174,4 & 1,090 & 3,02 & 4,25 \\
\hline
\end{tabular}

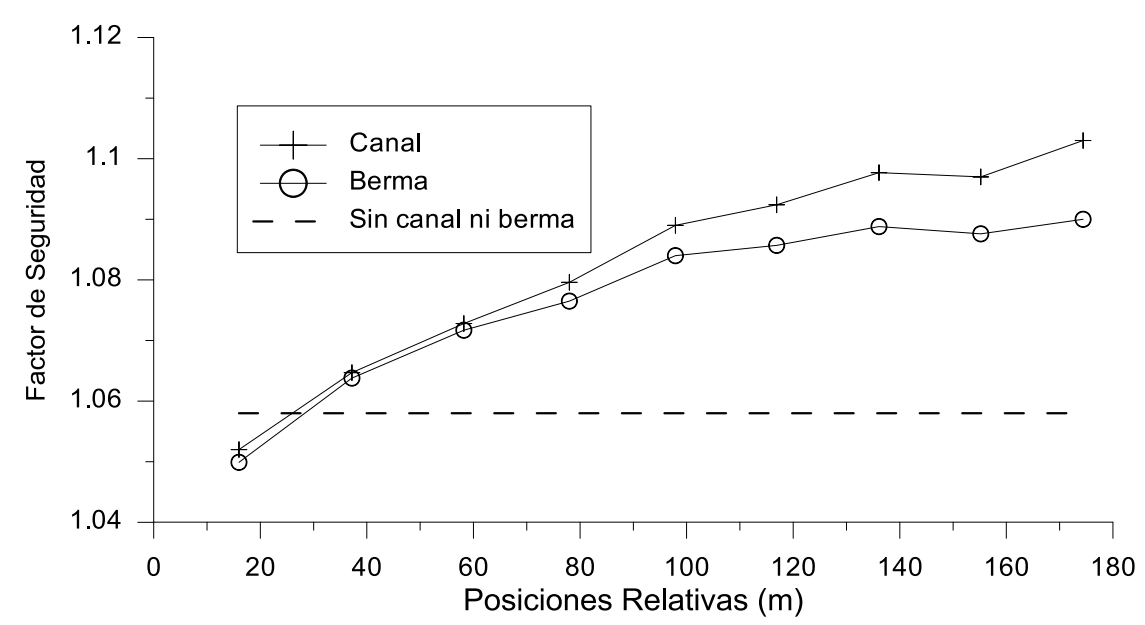

Figura 11. Factor de seguridad (pseudo-estático) respecto a la posición.

\subsubsection{Variación de las dimensiones del canal}

Con la finalidad de evaluar el efecto de las dimensiones del canal en el incremento de los FS en los análisis de estabilidad, se realizaron análisis complementarios de estabilidad por elementos finitos variando las dimensiones del canal en la ubicación 9, es decir, a 174,4 m de distancia del pie de la pila. Las dimensiones que se consideran como variables son la longitud $\mathrm{L}$ y la profundidad $\mathrm{H}$, permaneciendo constante la pendiente del talud $2 \mathrm{H}: 1 \mathrm{~V}$, además del espesor del material de baja permeabilidad es de $0,30 \mathrm{~m}$. En la Tabla 12 y Tabla 13 se presentan los resultados obtenidos.

CANAL

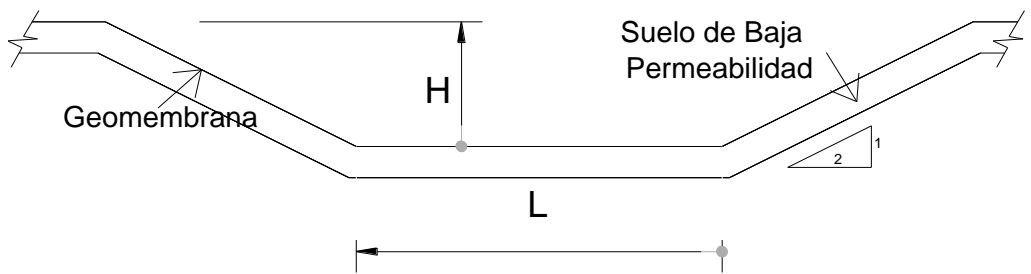

Figura 12. Variables de dimensiones en el canal. 
Tabla11. Dimensiones

\begin{tabular}{cc}
\hline $\mathrm{H}(\mathrm{m})$ & $\mathrm{L}(\mathrm{m})$ \\
\hline 0,75 & 3,50 \\
1,20 & 5,00 \\
1,65 & 6,50 \\
\hline
\end{tabular}

De este modo se logra los siguientes resultados de factor de seguridad (condiciones estáticas y pseudo-estáticas de carga).

Tabla12. FSestático de las diferentes combinaciones de las dimensiones de L y H.

\begin{tabular}{cccc}
\hline $\mathrm{H}(\mathrm{m})$ & 0,75 & 1,20 & 1,65 \\
$\mathrm{~L}(\mathrm{~m})$ & & & \\
\hline 3,50 & 1,724 & 1,740 & 1,743 \\
5,00 & 1,719 & 1,740 & 1,740 \\
6,50 & 1,728 & 1,738 & 1,742 \\
\hline
\end{tabular}

Tabla13. FSpseudo-estático de las diferentes combinaciones de las dimensiones de L y H.

\begin{tabular}{cccc}
\hline $\mathrm{H}(\mathrm{m})$ & 0,75 & 1,20 & 1,65 \\
$\mathrm{~L}(\mathrm{~m})$ & & & \\
\hline 3,50 & 1,095 & 1,103 & 1,094 \\
5,00 & 1,094 & 1,096 & 1,096 \\
6,50 & 1,094 & 1,093 & 1,095 \\
\hline
\end{tabular}

De acuerdo a estos resultados se concluye que no existen mayores incrementos del FS con el aumento de la longitud del canal; sin embargo, si se observa una tendencia del incremento del FS con el aumento de la profundidad independientemente de la longitud. 


\subsection{Análisis de superficies de falla}

3.3.1. Resultados de los análisis sin berma ni canal

(a)

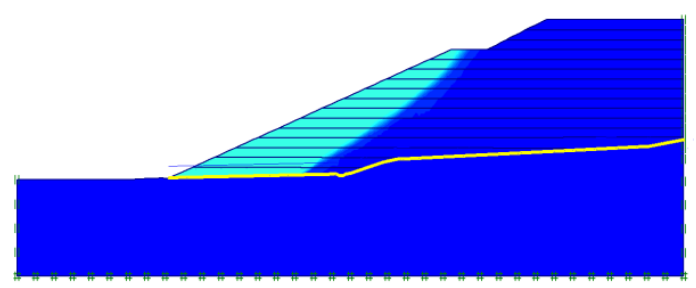

(b)

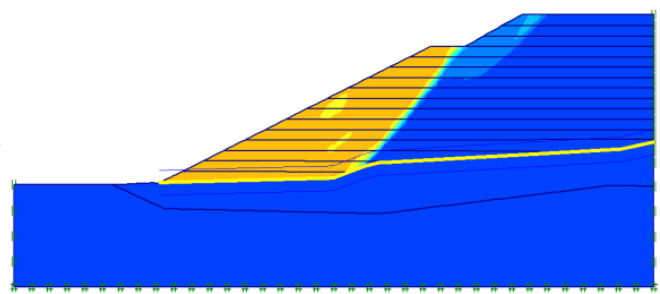

Figura 13. Superficie de falla en condiciones estáticas (a) y pseudo-estáticas (b).

\subsubsection{Resultados de los análisis con canal}

(a)

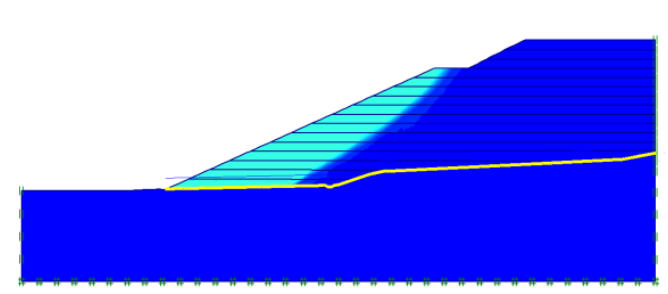

(b)

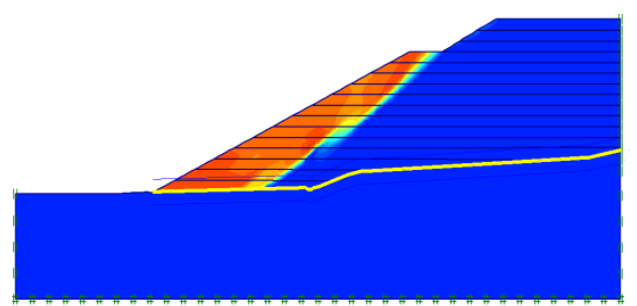

Figura 14. Superficie de falla en condiciones estáticas (a) y pseudo-estáticas (b).

Cuando se proyecta un canal y se analiza la estabilidad en condiciones estáticas y pseudo-estáticas, la superficie de falla en ambas condiciones resulta muy cercanas entre si, por tanto la cantidad de masa movilizada sería la misma.

\section{Esfuerzos}

Se presenta todas en condiciones pseudo-estáticas debido a que es las más crítica.
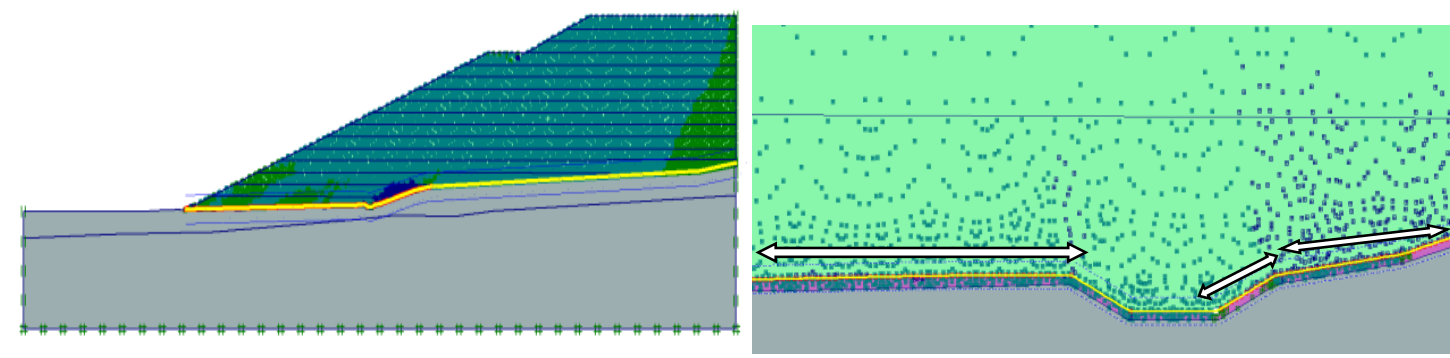

Figura 15. Puntos plásticos de Mohr Coulomb.

Los puntos plásticos de falla son los puntos en los cuales el estado de esfuerzos se encuentra ubicado en la envolvente de falla de Mohr Coulomb, los mismos que se pueden apreciar mediante flechas blancas en la figura anterior. Estos ocurren en la región de la interfase; 
sin embargo, la pendiente lateral izquierda del canal y la parte central no se ven afectados tanto como en el resto de la interfase.

En la Figura16 se muestra el incremento de desplazamiento debido solo a la aplicación de la carga pseudo-estática, se puede ver en la región señalada que una cara del canal tiende a contener los incrementos de desplazamiento, y probablemente esa sea una de las razones por la cual la superficie de falla tiende a acercarse hacia el talud.

(a)

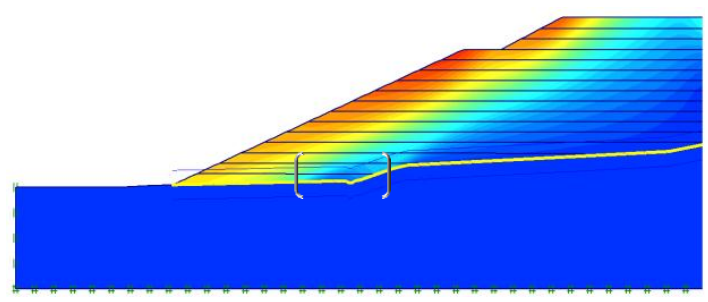

(b)

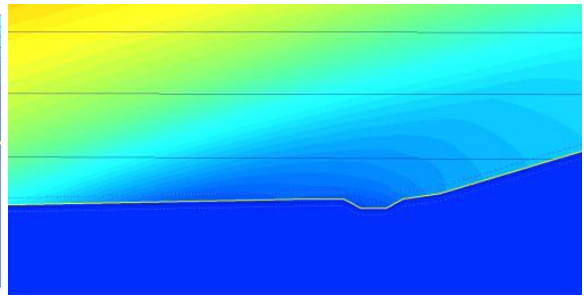

Figura 16. Incremento de desplazamiento debido solo a la carga pseudo-estática. En (a) se ve el esquema general y en (b) un detalle.

Lo anterior se podría comprobar cuando se proyectan dos canales a una distancia determinada y de este modo el incremento de desplazamiento se ve afectado de manera crítica tal y como se explicó anteriormente y la superficie de falla se acerca aún más hacia el talud, ver Figura17.

(a)

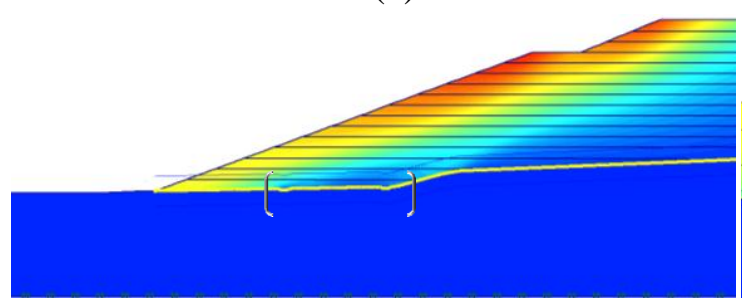

(b)

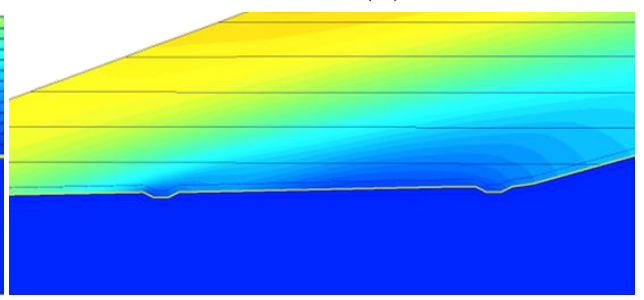

Figura 17. Incremento de desplazamiento debido solo a la carga pseudo-estática. En (a) se ve el esquema general y en (b) un detalle.

La figura anterior muestra la tendencia de los dos canales por tratar de contener el incremento de desplazamiento debido a la carga pseudo-estática. Entonces esto se traduce en la tendencia de la superficie de falla de acercarse aún más hacia el talud así como lo muestra la Figura18.

(a)

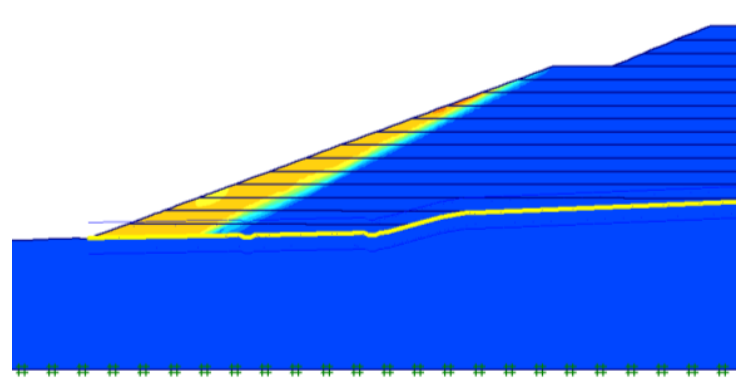

(b)

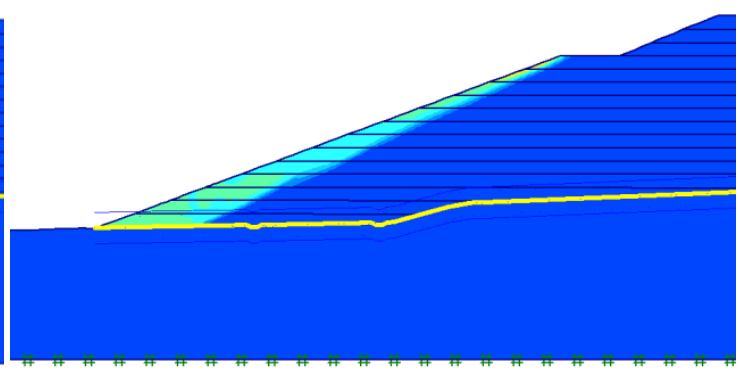

Figura 18. Superficie de falla en condiciones estáticas (a) y pseudo-estáticas (b). 
En la siguiente tabla se resume los resultados.

Tabla14. Resumen de resultados en FS y volumen movilizado debido a la superficie de falla para un canal y dos canales

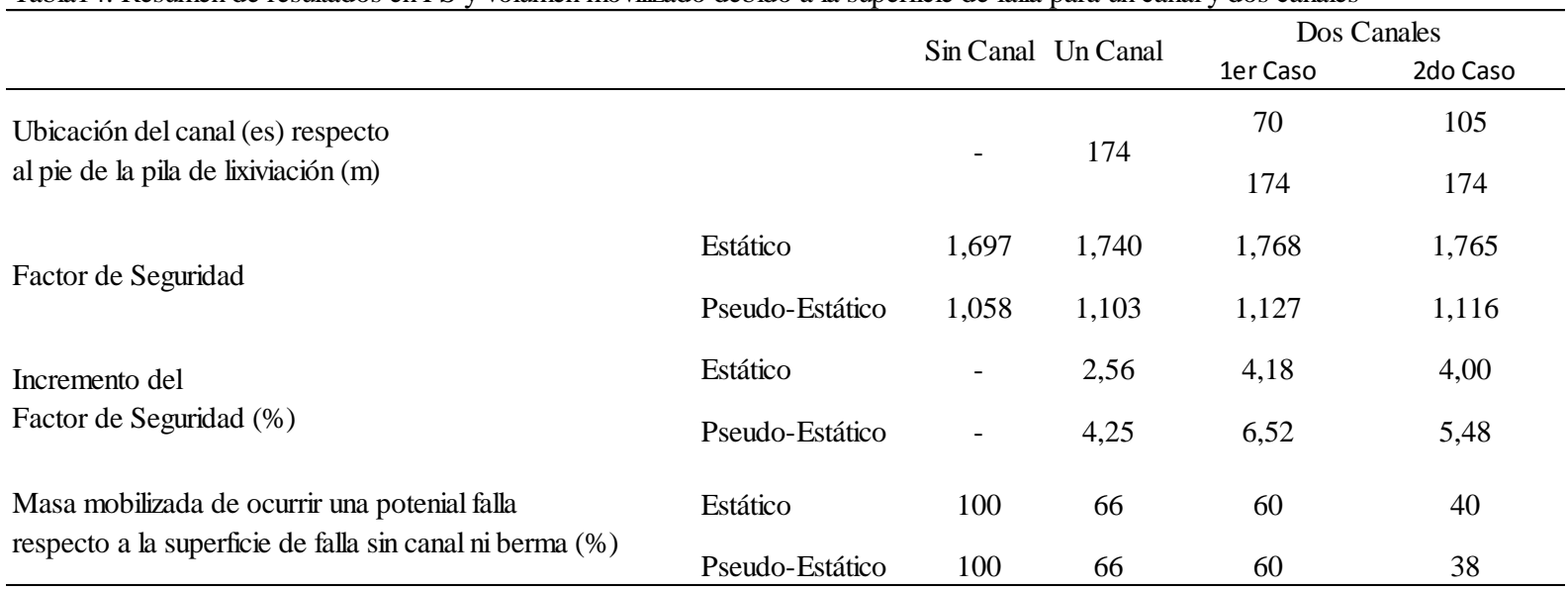

\subsection{Comparación de los análisis por equilibrio límite y elementos finitos}

En el análisis por elementos finitos y por equilibrio límite se encontró para los mismos materiales considerados con las mismas propiedades:

Tabla15. Valores de FS con ambos métodos y en las dos diferentes condiciones (Estáticas Y Pseudo-Estáticas).

\begin{tabular}{llcc}
\hline \multicolumn{1}{c}{$\begin{array}{c}\text { Condición } \\
\text { Analizada }\end{array}$} & \multicolumn{1}{c}{$\begin{array}{c}\text { Método de } \\
\text { Análisis }\end{array}$} & Sin Canal & Con Canal \\
\hline \multirow{2}{*}{ Estático } & Equilibrio Límite & 1,674 & 1,740 \\
& Elementos Finitos & 1,697 & 1,740 \\
\multirow{2}{*}{ Pseudo-Estático } & Equilibrio Límite & 1,085 & 1,148 \\
& Elementos Finitos & 1,058 & 1,103 \\
\hline
\end{tabular}

En general se podría decir a priori que el método de elementos finitos nos da como resultado un FS que tiende a ser menor que el de equilibrio límite; sin embargo, este resultado depende también del método de equilibrio límite usado, en este caso se usó el método de Spencer.

La Figura19 muestra las superficies de falla para el caso con y sin canal en condición estática y pseudo-estática de carga y se ve claramente que son muy cercanas, movilizando la misma cantidad de masa de mineral de ocurrir una potencial falla, independiente de que si hay o no canal, esto en el caso de analizar mediante equilibrio límite. Vía elementos finitos si existe gran diferencia entre superficies de falla. 
(a)

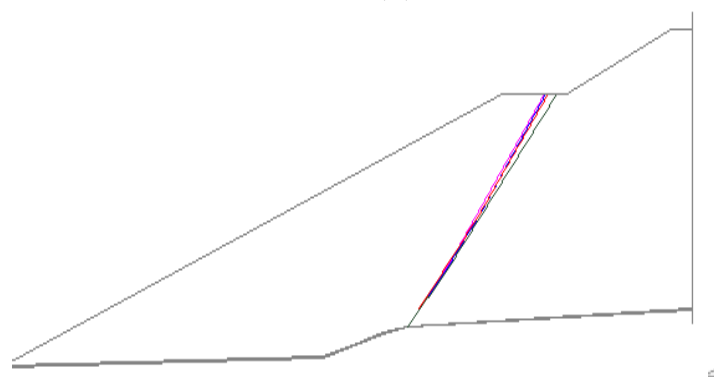

(b)

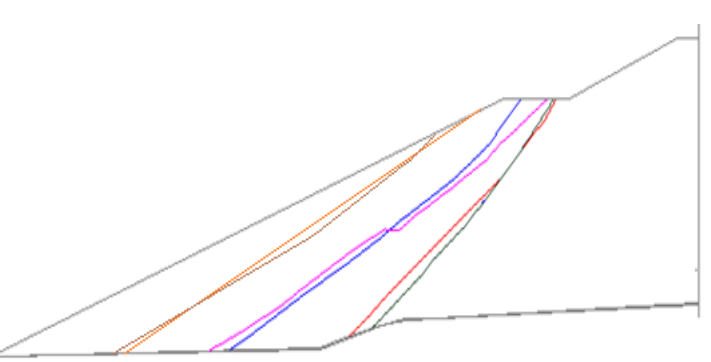

Figura 19. Superficies de falla y factores de seguridad por método de equilibrio límite (a) y elementos finitos (b).

Tabla16. Superficies de Falla y valores de FS con y sin canal por Equilibrio Límite.

\begin{tabular}{llll}
\hline & \multicolumn{1}{c}{$\begin{array}{c}\text { Condición } \\
\text { Analizada }\end{array}$} & FS & Leyenda \\
\hline Superficie de falla sin & Estático & 1,674 & \\
canal & Pseudo-Estático & 1,085 & \\
\hline \multirow{2}{*}{ Superficie de falla con } & Estático & 1,740 & \\
canal & Pseudo-Estático & 1,148 & \\
\hline
\end{tabular}

Tabla17. Superficies de Falla y valores de FS con y sin canal vía Elementos Finitos.

\begin{tabular}{llll}
\hline & \multicolumn{1}{c}{$\begin{array}{c}\text { Condición } \\
\text { Analizada }\end{array}$} & FS & Leyenda \\
\hline $\begin{array}{l}\text { Superficie de falla sin } \\
\text { canal }\end{array}$ & Estático & 1,697 \\
& Pseudo-Estático & 1,058 & \\
\hline \multirow{2}{*}{$\begin{array}{l}\text { Superficie de falla con } \\
\text { canal }\end{array}$} & Estático & 1,740 \\
\hline Superficie de falla con & Pseudo-Estático & 1,103 \\
Estático & 1,765 \\
\hline
\end{tabular}

\section{CONCLUSIONES}

Los análisis realizados permiten concluir que la mayor influencia sobre el FS de un talud de una pila de lixiviación es con la construcción de un canal de dimensiones estándar, en lugar de usar la estructura de una berma. Del mismo modo la superficie de falla se acerca hacia el talud movilizando una cantidad menor de masa cuando se emplea un canal e incrementando con ello el FS. Asimismo al proyectarse dos canales en lugar de uno se logra un mayor incremento en el FS y una reducción significativa de la masa en la superficie de falla. Adicionalmente, queda validado el procedimiento para el cálculo de estabilidad por equilibrio límite incluyendo bermas y/o canales; sin embargo, las superficies de falla encontradas no serían las más cercanas a lo real, lo cual estaría mejor representada utilizando el método de elementos finitos. El uso de un modelo más básico (Modelo constitutivo Mohr - Coulomb), aplicación 
del total de la carga debido al apilamiento del mineral en una sola fase de cálculo, obviar la existencia del suelo de baja permeabilidad tomando en cuenta el comportamiento de la interfase; se traduce en un ahorro significativo en tiempo de computo resultando cercano a la cuarta parte del tiempo empleado sin tener en consideración las simplificaciones indicadas.

\section{AGRADECIMIENTOS}

Se agradece a la Universidad Nacional de Ingeniería (Lima - Perú) que mediante el IIFIC me dio la oportunidad de acceder a un convenio con la empresa AUSENCO PERÚ que me brindó la información requerida. A mi asesor Denys Parra por su tiempo y su orientación, a mis compañeros por su asesoría y más importante a Dios y a mis Padres.

\section{REFERENCIAS}

[1] J.M. Duncan, Peter B., Kai S. Wong and Philip Mabry "Strength, Stress - Strain And Bulk Modulus Parameters For Finite Element Analysis Of Stresses And Movements In Soil Masses", 1980.

[2] T. Schanz "The Hardening Soil Model: formulation and verification", Laboratory of soil mechanics, Bauhaus-University Weimar, Germany, 1999.

[3] Manual Del Software PLAXIS, 2004.

[4] Andrés N. L., Javier C. T., Edwin R. B., "Determinación De Parámetros Para Los Modelos Elasto-plásticos Mohr-Coulomb Y Hardening Soil En Suelos Arcillosos”, Universidad Militar Nueva Granada, Bogotá, 2009.

[5] R. E. Goodman, "Introduction to Rock Mechanics” second Edition, 1989.

[6] D.V. Griffiths, P.A. Lane - Colorado School, Golden, Colorado, U.S.A. - "Slope Stability Analysis By Finite Elements", 1999. 